\title{
Variations in hospital standardised mortality ratios (HSMR) as a result of frequent readmissions
}

Wim F van den Bosch ${ }^{1 *}$, Peter Spreeuwenberg ${ }^{2}$ and Cordula Wagner ${ }^{2,3}$

\begin{abstract}
Background: We investigated the impact that variations in the frequency of readmissions had upon a hospital's standardised mortality ratio (HSMR). An adapted HSMR model was used in the study. Our calculations were based on the admissions of 70 hospitals in the Netherlands during the years 2005 to 2009.

Methods: Through a retrospective analysis of routinely collected hospital data, we calculated standardised inhospital mortality ratios both by hospital and by diagnostic group (H/SMRs) using two different models. The first was the Dutch 2010 model while the second was the same model but with an additional adjustment for the readmission frequency. We compared H/SMR outcomes and the corresponding quality metrics in order to test discrimination (c-statistics), calibration (Hosmer-Lemeshow) and explanatory power (pseudo- $R^{2}$ statistic) for both models.
\end{abstract}

Results: The SMR outcomes for model 2 compared to model 1, varied between $-39 \%$ and $+110 \%$. On the HSMR level these variations ranged from $-12 \%$ to $+11 \%$. There was a substantial disagreement between the models with respect to significant death on the SMR level as well as the HSMR level $(\sim 20 \%)$. All quality metrics comparing both models were in favour of model 2. The susceptibility to adjustment for readmission increased for longer review periods.

Conclusions: The 2010 HSMR model for the Netherlands was sensitive to adjustment for the frequency of readmissions. A model without this adjustment, as opposed to a model with the adjustment, produced substantially different HSMR outcomes. The uncertainty introduced by these differences exceeded the uncertainty indicated by the $95 \%$ confidence intervals. Therefore an adjustment for the frequency of readmissions should be considered in the Netherlands, since such a model showed more favourable quality metric characteristics compared to a model without such an adjustment. Other countries could well benefit from a similar adjustment to their models. A review period of the data collected over the last three years, at least, is advisable.

\section{Background}

Hospital standardised mortality ratios (HSMRs) are widely used as an indicator to assess and improve the quality of care. HSMRs have been calculated in the UK, USA, Canada, Australia and the Netherlands [1]. HSMR outcomes fluctuate around 100. For example in the UK the outcomes range from 72 to 118 and in the Netherlands from 62 to 142 [1,2]. The Dutch HSMR model was developed by the Dr. Foster Intelligence Unit in London in co-operation with Kiwa Prismant in the Netherlands. The model adjusts for patient casemix factors including age, sex and diagnostic group. It is based upon the

\footnotetext{
* Correspondence: w.bosch@antoniusziekenhuis.nl

'St. Antonius Hospital, P.O. Box 2500, 3430, EM Nieuwegein, the Netherlands Full list of author information is available at the end of the article
}

national medical registration data (LMR). In October 2010 the 'Dutch Hospital Data' group (DHD) distributed the hospital specific, 'H/SMR report 2007-2009 with detailed information on diagnostic groups and patient categories', among Dutch hospitals. In this way, each hospital obtained an insight into their own standardised mortality ratios (SMRs) for 50 diagnostic groups and had the opportunity to work on improving patient safety.

HSMRs are also made publicly available in countries, for example in the UK's '2010 Dr Foster Hospital Guide'[2]. However questions have been raised about how reliable, valid and applicable the model really is [3-10]. It is still not clear to what degree the current HSMR and SMR outcomes are attributable to quality of care and can be used to make a meaningful comparison between hospitals.

\section{(Ciomed Central}


Bottle et al claim however that variations in adjustment methods have a limited impact on the hospitals position relative to funnel plot control limits [11].

One type of potential distortion in establishing comparable HSMRs is caused by the failure to adjust for variations in readmission frequencies. Van den Bosch, et al [10] have demonstrated that the Dutch 2010 HSMR model favoured hospitals with relatively many frequently readmitted patients, regardless of disease, compared to hospitals with many patients who are not frequently readmitted. They were, however, not able to quantify the effect of this upon the HSMR, because the study was restricted to only six hospitals. In a new study more data was made available enabling us to quantify the possible effect in the Netherlands. We were able to compare the HSMR outcomes of the current HSMR model to an alternative model with an additional adjustment for readmission frequency. In essence we asked: Does the new model represent an improvement in the current model, based on c-statistics, goodness of fit and explanatory power? And if so, to what extent do HSMR and SMR outcomes differ, comparing model 1 to model 2 ?

\section{Methods}

\section{Setting}

For the purpose of this model analysis the DHD group gave its formal permission to use an anonymised LMR dataset from 89 Dutch hospitals covering the years 2005 to 2009. Nineteen hospitals were excluded from the calculation because the quality of their patient registration data was insufficient.

This study excluded any experimental research, either on human or animal subjects.

\section{The HSMR model used in this study}

We performed HSMR calculations using two models, shown as model 1 and model 2.

For model 1, we used the Dr Foster model [1], applied in the Netherlands in 2010. This Dutch model included clinical admissions only, so no day cases. Furthermore only inhospital deaths were counted. There are differences between the Dutch and the UK model. The Dutch model used 50 Clinical Classifications Software (CCS) groups based on an ICD-9 coding, whereas the UK used 56 CCS groups based on ICD-10 coding, of which 42 groups were the same as in the Dutch model. Furthermore, the UK model adjusted for palliative care and for the number of previous emergency admissions within one year. The Dutch model did not adjust for either of these.

\section{HSMR in brief}

The HSMR ('hospital standardised mortality ratio') is the ratio between the observed number of in-hospital deaths and the predicted number of deaths, determined by comparing the patient casemix with the national average. The outcome is standardised around 100. A value above 100 indicates higher mortality than average, below 100, lower mortality. If the calculation is applied to one of the 50 diagnostic groups, then we speak of the SMR ('standardised mortality ratio').

The Dutch HSMR model of 2010 adjusts for the following casemix properties: year of discharge, sex, age at admission, admission type, comorbidity (charlsonindex), social deprivation, month of admission, source of referral, diagnostic group, and, in part, for the casemix on the primary diagnostic level.

Each diagnostic group is composed of a number of underlying diseases: ICD-9 codes (International Classification of Diseases Ninth edition) as determined by the Clinical Classification Software (CCS). This tool clusters various ICD-9 diagnoses into a manageable number of meaningful categories [12], indicated as 'CCS diagnostic groups'. The selected CCS diagnostic groups have a relatively high mortality and together cover over $80 \%$ of the total number of hospital deaths.

For model 2, we took model 1 and added an adjustment for the frequency of readmission, as described by Van den Bosch WF, et al [10]. The authors demonstrated that frequently admitting patients was associated with lower mortality ratios per admission, for which an additional correction would be needed. In their publication the patients were grouped into 'patient view' classes $\mathrm{P}(\mathrm{m})$. We have also applied this to our study. Here the admission frequency $m$ was equal to the number of times a patient was admitted to the same hospital during the five-year study period.

For example, a patient who was admitted ten times during the five-year period to hospital $\mathrm{X}$, to be treated for one or more diseases, possibly with different diagnoses, was part of the patient view class $\mathrm{P}(10)$ and not part of any other patient view class. This patient contributed ten admissions to $\mathrm{P}(10)$ which were all accounted for in the regression calculation.

We were able to retrieve the number of times that a patient was admitted through the unique patient identification number that all of the 70 hospitals included were using. We have grouped the patient view classes into eight categories: $\mathrm{m}=1, \mathrm{~m}=2, \mathrm{~m}=3, \mathrm{~m}=4, \mathrm{~m}=5-6$, $\mathrm{m}=7-9, \mathrm{~m}=10-20$ and $\mathrm{m}>20$. This is in order to limit the number of categories for the regression calculation and to avoid categories becoming too small.

\section{Agreement, or otherwise of the two models}

We have calculated to what extent both models did agree, or did not agree, as follows:

1. Relative change. To what extent did $\mathrm{SMR}_{\text {model } 1}$ differ from $\mathrm{SMR}_{\text {model } 2}$ per CCS diagnostic group, per hospital? Using the formula $\mathrm{SMR}_{\text {delta }}=\left(\mathrm{SMR}_{\text {model2 }} / \mathrm{SMR}_{\text {model1 }}-1\right)$ 
* $100 \%$, we calculated the shifts for all of the 3500 SMRs (50 diagnostic groups times, 70 hospitals), occurring when changing from model 1 to model 2 . We represented these in a frequency distribution. In a similar way we have calculated and represented the 70 shifts that occurred for the HSMRs of the 70 hospitals. We also compared all pairs of death predictions of model 1 and model 2 per admission by calculating regressions coefficients $\left(R^{2}\right)$ on the SMR level and the HSMR level. These metrics were used as a measure of statistical 'distance' between both models.

2. Significance scores of SMRs and HSMRs. We determined per SMR how many hospitals with a significantly high SMR score according to model 1 turned out not to be significantly high according to model 2 . And vice versa, in other words: how often a significantly high SMR score in model 2 turned out not to be a significantly high score in model 1 . In a similar way we calculated these differences on the hospital level for HSMRs.

\section{Quality metrics of the two models}

We have calculated and compared the quality metrics of the models with respect to:

1. Discrimination, expressed in 'c-statistics' on the SMR level and on the HSMR level. This statistical measure indicates how well a regression model is able to predict mortality. Each predicted outcome per admission is compared to the observed outcome: died or survived. A c-statistic of 0.5 has no predictive value: $50 \%$ right, $50 \%$ wrong. Values above 0.75 suggest good discrimination. A value of 1 is perfect. The overall c-statistic for the Dutch HSMR (model 1) scored above 0.85 .

2. Calibration, according to Hosmer and Lemeshow. This represents a statistical test for goodness-of-fit that is frequently used in risk prediction models. The test assesses whether or not the observed event rates match expected event rates in subgroups of the model population. The test specifically identifies subgroups as the deciles of fitted risk values. Models in which expected and observed event rates in subgroups are similar, are called well-calibrated [13].

3. Explanatory power, using the pseudo $\mathrm{R}^{2}$ statistic according to the 'Nagelkerke R square', in order to assess the degree to which the additional adjustment for readmission changes the unexplained variance in the data.

\section{Sensitivity of the HSMR model to adjustment for readmission}

We have investigated to what extent the model was sensitive to variations in the length of the period under review. We took three scenarios: a period of review of one year (2009), two years (2008-2009) and five years (2005-2009). We then examined the statistical distance between model 1 and model 2, and the three model quality metrics on the HSMR level.

\section{Results}

We analysed the data from 89 hospitals of which 19 were excluded because they had an incomplete registration of the patient identification numbers. Therefore, we included 70 hospitals for the regression calculation, a total of 2494613 admissions.

\section{Agreement between the two models}

Figure 1 shows the frequency distribution of the relative changes of the 3500 SMRs, in going from model 1 to model 2. Similarly the changes of the 70 HSMRs of the hospitals are shown in Figure 2. The SMR changes ranged from $-39 \%$ to $+110 \%$ and the HSMR changes ranged from $-12 \%$ to $+11 \%$. The standard deviation of the frequency distribution of the changes amounted to $12.2 \%$ (roughly 12 SMR points) for the SMRs and to $4.1 \%$ (roughly 4 HSMR points) of the HSMRs.

Table 1 shows the agreement that we found between model 1 and model 2, expressed as a statistical distance per SMR. The $\mathrm{R}^{2}$ coefficients varied from 0.60 (COPD) to 0.95 (intracranial injury). For the entire model the coefficient amounted to 0.86 .

Finally, we calculated per SMR for how many hospitals a significantly high SMR score changed to not significantly high from model 1 to model 2 and vice versa; see Table 1. Model 1 indicated 328 SMRs in total as "higher than expected" of which 64 were not indicated by model 2 (20\%). On the other hand, model 2 indicated 313 SMRs in total as "higher than expected" of which 49 were not indicated by model 1 (16\%). Across hospitals, model 1 indicated that 23 hospitals recorded a higher than

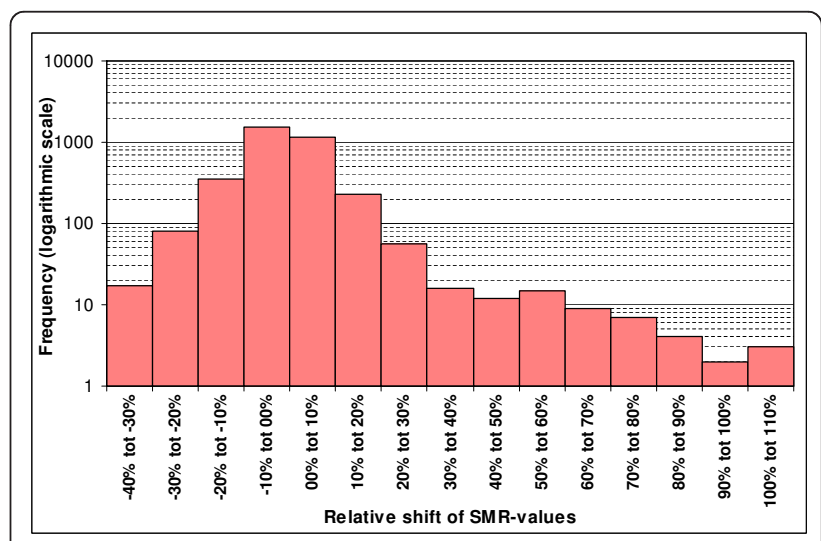

Figure 1 Frequency distribution of relative changes of all 3500 SMRs when changing from model 1 (Dr Foster model) to model 2 (model with additional adjustment for readmission frequency). * Standard deviation amounts to $12.2 \%$. 


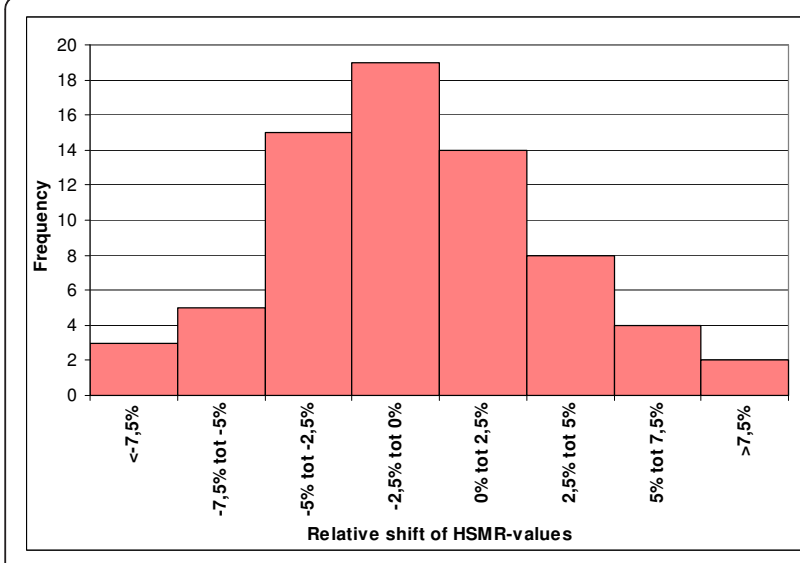

Figure 2 Frequency distribution of relative changes of the 70 HSMRs when changing from model 1 (Dr Foster model) to model 2 (model with additional adjustment for readmission frequency). * Standard deviation amounts to $4.1 \%$.

expected HSMR of which four were not indicated by model 2 (17\%). And, alternatively, model 2 indicated that 23 hospitals were having a higher than expected HSMR of which four were not indicated by model 1 (17\%).

\section{H/SMR model quality metrics}

For both models the 'c-statistic' per CCS diagnostic group was calculated, see Table 1 . Model 2 scored a higher c-statistic compared to model 1 in 47 groups out of 50. For eighteen of these groups the scores of model 2 exceeded the scores of model 1 by between 0.03 and 0.08 . Model 1 had one diagnostic group for which the c-statistic was at least 0.03 better compared to model 2. The c-statistic of the HMSR across all hospitals amounted to 0.852 for model 1 and to 0.867 for model 2 (favourable).

The results of the goodness-of-fit test are shown in Table 2, expressed in the quality metrics of the Hosmer and Lemeshow test. It shows that model 2 was better calibrated compared to model 1: 7434 mismatches (5.75\% of the total deaths) for model 1 compared to 5474 (4.23\%) mismatches for model 2.

Finally the overall explanatory power expressed in pseudo $\mathrm{R}^{2}$ statistics were calculated. These amounted to 0.259 for model 1 versus 0.282 for model 2 and so favourable to model 2 .

\section{Sensitivity of the model to the review period of adjustment}

The overall agreement between models 1 and 2 varied, depending on the review period, from 0.92 (one year) to 0.89 (two years) to 0.86 (five years). So a longer review period corresponded with an increasing divergence between the two models.

Table 3 shows how the overall model quality metrics varied for the three review periods. A longer review period corresponded to an increase of model quality metric performance.

\section{Discussion}

How should we interpret the differences in model outcomes?

In this study we have calculated and compared the $\mathrm{H} /$ SMR outcomes of two models. The disagreement between the two models was substantial, according to the relative changes in HSMRs and SMRs.

The standard deviation of the frequency distribution of HSMR-change amounted to 4 HSMR points, which was substantial compared to the standard deviation of the HSMR-frequency distribution amounting to 14 HSMR points. Moreover, these shifts introduced an uncertainty into the HSMR outcomes that exceded the uncertainty due to chance as indicated by the $95 \%$ confidence intervals (CIs): The $95 \%$ CI of a HSMR equalled $[-2 \sigma, 2 \sigma]$. On average for the 70 hospitals over five years, the standard deviation $\sigma$ of the CI distribution equalled 2.5 HSMR points.

With respect to the SMRs: the statistical 'distance' between SMRs of model 1 and model 2 expressed in $\mathrm{R}^{2}$ coefficients, varied from 0.60 to 0.95 . Low scores indicated susceptibility to adjustment for readmissions. On average chronic diseases scored lower compared to acute diseases as to be expected since chronic diseases in particular often result in readmissions. There was no particular peak among any of the main diagnostic groups. The major diagnostic groups like cancer and heart and lung diseases all showed substantial disagreement between model 1 and model 2 due to adjustment for readmission. The results also showed disagreement between the models in designating hospitals as having higher than expected H/SMRs. The percentages of disagreement varied from $16 \%$ to $20 \%$.

Bottom-line: all differences in H/SMR outcomes that we found between the two models, cannot be attributed to differences in the quality of care neither to 'chance'. They have to be attributed to the difference in the type of model, because this is the only distinction applied in producing the results from the same set of admissions. This implies that publicised good and bad practices, linked to hospital rankings based on HSMRs, can differ substantially depending on the choice of model.

\section{How do readmissions affect H/SMRs and how do they occur?}

We asked: how did readmissions impact the H/SMR, how did readmissions occur and why did hospitals differ in this respect?

For example: endlessly readmitting the same patient in the same hospital continuously increased the denominator (predicted death) of the calculated HSMR for that 
Table 1 A comparison of SMR outcomes of model 1 and model 2

\begin{tabular}{|c|c|c|c|c|c|c|c|}
\hline \multirow[t]{2}{*}{$\begin{array}{l}\text { CCS } \\
\text { code }\end{array}$} & \multirow[t]{2}{*}{ CCS-diagnostic group title } & \multirow[t]{2}{*}{$\begin{array}{l}\text { Number of } \\
\text { admissions }\end{array}$} & \multirow{2}{*}{$\begin{array}{c}\text { Distance } \\
\text { between } \\
\text { model } 1 \\
\text { and } 2\left(R^{2}\right)\end{array}$} & \multicolumn{2}{|c|}{$\begin{array}{l}\text { Number of hospitals with } \\
\text { SMR significantly high? }\end{array}$} & \multicolumn{2}{|c|}{ c-statistic for } \\
\hline & & & & $\begin{array}{c}\text { Model 1: } \\
\text { no } \\
\text { model 2: } \\
\text { yes }\end{array}$ & $\begin{array}{c}\text { Model 1: } \\
\text { yes } \\
\text { model 2: } \\
\text { no }\end{array}$ & $\begin{array}{l}\text { Model } \\
1\end{array}$ & $\begin{array}{c}\text { Model } \\
2\end{array}$ \\
\hline 2 & Septicemia (except in labour) & 14647 & 0.83 & 2 & 1 & 0.760 & 0.783 \\
\hline 12 & Cancer of oesophagus & 9703 & 0.74 & 1 & 3 & 0.730 & 0.763 \\
\hline 13 & Cancer of stomach & 13945 & 0.75 & 0 & 2 & 0.733 & 0.779 \\
\hline 14 & Cancer of colon & 45116 & 0.77 & 2 & 5 & 0.773 & 0.814 \\
\hline 15 & Cancer of rectum and anus & 22246 & 0.72 & 1 & 3 & 0.771 & 0.817 \\
\hline 17 & Cancer of pancreas & 9950 & 0.76 & 1 & 0 & 0.685 & 0.704 \\
\hline 19 & Cancer of bronchus, lung & 76714 & 0.75 & 4 & 6 & 0.792 & 0.822 \\
\hline 24 & Cancer of breast & 64126 & 0.74 & 2 & 1 & 0.916 & 0.850 \\
\hline 29 & Prostate cancer & 24092 & 0.76 & 3 & 1 & 0.886 & 0.865 \\
\hline 32 & Cancer of bladder & 44288 & 0.71 & 1 & 0 & 0.872 & 0.874 \\
\hline 38 & Non-Hodgkin's lymphoma & 19413 & 0.69 & 0 & 5 & 0.802 & 0.841 \\
\hline 39 & Leukaemias & 12466 & 0.77 & 0 & 1 & 0.781 & 0.820 \\
\hline 42 & Secondary malignancies & 69303 & 0.77 & 5 & 4 & 0.748 & 0.770 \\
\hline 44 & $\begin{array}{l}\text { Neoplasms unspec nature or uncertain } \\
\text { behaviour }\end{array}$ & 18982 & 0.63 & 2 & 1 & 0.805 & 0.840 \\
\hline 50 & Diabetes mellitus with complications & 36837 & 0.74 & 1 & 0 & 0.824 & 0.846 \\
\hline 55 & Fluid and electrolyte disorders & 29705 & 0.81 & 0 & 1 & 0.795 & 0.824 \\
\hline 59 & Deficiency and other anaemia & 48896 & 0.61 & 0 & 1 & 0.756 & 0.802 \\
\hline 85 & Coma, stupor and brain damage & 4286 & 0.92 & 0 & 0 & 0.801 & 0.815 \\
\hline 96 & Heart valve disorders & 30708 & 0.77 & 1 & 2 & 0.775 & 0.788 \\
\hline 100 & Acute myocardial infarction & 91802 & 0.72 & 1 & 1 & 0.740 & 0.781 \\
\hline 101 & $\begin{array}{l}\text { Coronary atherosclerosis and other heart } \\
\text { disease }\end{array}$ & 250183 & 0.66 & 1 & 2 & 0.784 & 0.805 \\
\hline 103 & Pulmonary heart disease & 26937 & 0.78 & 0 & 1 & 0.754 & 0.776 \\
\hline 106 & Cardiac dysrhythmias & 174923 & 0.72 & 1 & 2 & 0.828 & 0.860 \\
\hline 107 & Cardiac arrest and ventricular fibrillation & 8118 & 0.89 & 2 & 2 & 0.739 & 0.764 \\
\hline 108 & Congestive heart failure, non-hypertensive & 108541 & 0.63 & 4 & 1 & 0.637 & 0.702 \\
\hline 109 & Acute cerebrovascular disease & 102295 & 0.86 & 3 & 0 & 0.744 & 0.761 \\
\hline 114 & Peripheral and visceral atherosclerosis & 44090 & 0.94 & 1 & 3 & 0.908 & 0.914 \\
\hline 115 & Aortic, peripheral and visceral artery aneurysms & 26538 & 0.93 & 1 & 1 & 0.871 & 0.887 \\
\hline 116 & $\begin{array}{l}\text { Aortic \& peripheral arterial embolism or } \\
\text { thrombosis }\end{array}$ & 32881 & 0.75 & 1 & 1 & 0.882 & 0.892 \\
\hline 117 & Other circulatory disease & 20452 & 0.80 & 0 & 1 & 0.849 & 0.862 \\
\hline 122 & Pneumonia & 128867 & 0.74 & 2 & 0 & 0.753 & 0.785 \\
\hline 127 & COPD and bronchiectasis & 82898 & 0.60 & 0 & 2 & 0.701 & 0.761 \\
\hline 129 & Aspiration pneumonitis & 4485 & 0.79 & 1 & 1 & 0.644 & 0.688 \\
\hline 130 & Pleurisy, pneumothorax, pulmonary collapse & 24798 & 0.78 & 0 & 0 & 0.812 & 0.827 \\
\hline 133 & Other lower respiratory disease & 99460 & 0.77 & 1 & 0 & 0.854 & 0.866 \\
\hline 145 & Intestinal obstruction without hernia & 34802 & 0.76 & 1 & 1 & 0.813 & 0.836 \\
\hline 146 & Diverticulosis and diverticulitis & 35917 & 0.70 & 0 & 0 & 0.814 & 0.828 \\
\hline 149 & Biliary tract disease & 135587 & 0.80 & 1 & 0 & 0.894 & 0.899 \\
\hline 150 & Liver disease, alcohol-related & 5133 & 0.69 & 0 & 2 & 0.678 & 0.758 \\
\hline 151 & Other liver diseases & 14584 & 0.80 & 1 & 0 & 0.771 & 0.807 \\
\hline 153 & Gastrointestinal haemorrhage & 37229 & 0.71 & 0 & 1 & 0.738 & 0.764 \\
\hline 155 & Other gastrointestinal disorders & 45487 & 0.91 & 0 & 0 & 0.907 & 0.908 \\
\hline 157 & Acute and unspecified renal failure & 8804 & 0.73 & 0 & 0 & 0.723 & 0.760 \\
\hline
\end{tabular}


Table 1 A comparison of SMR outcomes of model 1 and model 2 (Continued)

\begin{tabular}{|c|c|c|c|c|c|c|c|}
\hline 158 & Chronic renal failure & 14570 & 0.75 & 0 & 2 & 0.846 & 0.872 \\
\hline 159 & Urinary tract infections & 62382 & 0.77 & 0 & 0 & 0.817 & 0.836 \\
\hline 226 & Fracture of neck of femur (hip) & 74557 & 0.73 & 0 & 2 & 0.719 & 0.732 \\
\hline 233 & Intracranial injury & 56535 & 0.95 & 0 & 1 & 0.939 & 0.941 \\
\hline 237 & Complication of device, implant or graft & 76079 & 0.69 & 0 & 0 & 0.821 & 0.833 \\
\hline 238 & $\begin{array}{l}\text { Complications surgical procedures or medical } \\
\text { care }\end{array}$ & 67106 & 0.76 & 0 & 0 & 0.827 & 0.833 \\
\hline 249 & Shock & 3150 & 0.80 & 1 & 0 & 0.716 & 0.733 \\
\hline & Total all CCS diagnostic groups & 2494613 & 0,86 & 49 & 64 & 0.852 & 0.867 \\
\hline
\end{tabular}

* Per CCS diagnostic group: number of admissions; statistical distance between model 1 and model 2; number of hospitals with significance differences of SMRs comparing model 1 to model 2, c-statistics for both models. The table includes results of 70 Dutch hospitals over the period $2005-2009$

hospital. We have observed numerous cases where the contribution to the denominator on behalf of a single patient over the years aggregated to numerical values of 3 to 4 . However a patient could only die once and so any patient could maximally contribute a numerical value of 1 to the numerator. Consequently this effect lowered the HSMR ratio and favoured hospitals which had many frequently readmitted patients.

There are many types of mechanisms that explain the variation in the frequency of clinical readmission. These could have been administrative in nature, for example differences in administrating chemotherapy [10]. There were also many systematic differences in treatment practice. For example one hospital applied and recorded a different number of clinical admissions than another hospital did for the same combination of diagnosis and treatment applied to the same patient. Differences also occurred as a consequence of transferring patients back and forth. Poor care during the initial admission may also have triggered readmissions later. As a consequence all these examples of increased readmissions were being 'rewarded' by model 1 while model 2 was correcting for this phenomenon.

Table 2 Contingency table with Hosmer and Lemeshow test for goodness-of-fit

\begin{tabular}{ccccc}
\hline & \multicolumn{2}{c}{ Died model $\mathbf{1}$} & \multicolumn{2}{c}{ Died model 2 } \\
\cline { 2 - 5 } Decile & Observed & Expected & Observed & Expected \\
\hline 1 & 138 & 268 & 134 & 231 \\
2 & 498 & 786 & 469 & 677 \\
3 & 1057 & 1464 & 997 & 1295 \\
4 & 1888 & 2485 & 1721 & 2197 \\
5 & 3609 & 4038 & 3002 & 3544 \\
6 & 6181 & 6399 & 5299 & 5599 \\
7 & 10486 & 10026 & 8999 & 8843 \\
8 & 16797 & 15578 & 14890 & 14337 \\
9 & 28064 & 26026 & 27112 & 25084 \\
10 & 60625 & 62273 & 66720 & 67535 \\
\hline \multicolumn{2}{c}{ Percentage mismatch } & $5,75 \%$ & & $4,23 \%$ \\
\hline
\end{tabular}

The analysis showed that the combined effect of all these mechanisms was not restricted to some of the diagnostic groups, but had an impact upon most of them. Clearly the HSMR model was susceptible to an adjustment for the frequency of readmissions in the way we have defined it.

The analysis also showed that reducing the review period from five to two years and from two to one year, resulted in a substantial reduction of this susceptibility. This phenomenon may be explained as follows: It may take several years before the readmissions of a patient constitute a substantial sequence of admissions. Furthermore a sequence might also have started long before the analysis period started. In these cases apparently one year was not a sufficiently long time period to capture sequences with a substantial amount of admissions. By looking at various years these sequences became better visible.

\section{Choice of model: consequences for usage of H/SMR as indicator}

We considered which of the two models would be more favourable to use and why. Since the model characteristics with regard to discrimination, calibration and

Table 3 Comparison of HSMR model quality metrics of model 1 to model 2 for various lengths of review period

\begin{tabular}{lllll}
\hline & Model 1 & Model 2 & Difference & In favour of \\
Overall c-statistic & & & & \\
\hline 1 year period & 0.857 & 0.866 & 0.009 & model 2 \\
2 year period & 0.856 & 0.865 & 0.009 & model 2 \\
5 year period & 0.852 & 0.867 & 0.015 & model 2 \\
\hline $\begin{array}{llll}\text { \% decile match hosmer } \\
\text { lemeshow test }\end{array}$ & $93.85 \%$ & $94.13 \%$ & $0.28 \%$ & model 2 \\
\hline 1 y period & $93.82 \%$ & $94.41 \%$ & $0.59 \%$ & model 2 \\
2 year period & $94.25 \%$ & $95.77 \%$ & $1.52 \%$ & model 2 \\
\hline year period & & & model 2 \\
\hline Nagelkerke R square & & & & model 2 \\
\hline year period & 0.269 & 0.285 & 0.016 & model 2 \\
\hline year period & 0.266 & 0.284 & 0.018 & \\
\hline
\end{tabular}


explanatory power all were in favour of model 2, we recommend using model 2 , although this preference does not necessarily invalidate model 1 . HSMR models in other countries may need a similar additional adjustment. The impact of readmissions on the current HSMR in the UK is not clear. The UK model adjusts for readmissions for emergency cases. However for nonemergency cases the model does not adjust for readmissions. Moreover, admissions with different primary diagnoses are not counted as readmissions as well. Furthermore the UK model is restricted to a maximum review period of one year, which is too short for the readmission effect to become visible as demonstrated in our study.

A more fundamental issue than the choice of model however, is the fact that two comparable models, such as model 1 and model 2, may deliver such divergent outcomes. Since there is no 'gold standard' one cannot state that the one is false and the other one is true. This finding reveals an uncertainty in HSMR outcomes that exceeds the amount of uncertainty introduced by chance expressed in $95 \%$ confidence intervals over the five-year period.

HSMRs and SMRs are increasingly used by physicians to improve the quality of care in their hospitals. In particular diagnostic groups with higher than expected mortality are the subjects of investigation. However, in a lot of cases, the designated significance of these groups may depend on the regression model used, not only on the confidence intervals. The same is applicable for the current HSMR scores in the Netherlands. Physicians and hospital managers should be aware of this phenomenon.

\section{Limitations of the study}

A limitation of this study was the fact that we had to exclude 19 hospitals and so the HMSR calculation was based on roughly $80 \%$ of the Dutch hospitals. Nevertheless, we think the results of our study were representative of the Netherlands and have clearly demonstrated the impact upon the HSMR of not adjusting for frequency of readmissions.

Another limitation was the fact that the Dutch HSMR only accounts for in-hospital mortality and not for 30day mortality, which may favour hospitals with shorter lengths of stay [14]. However since the effects, demonstrated in our study, particularly concerned the phenomenon of the frequently returning patient, and so no 30 days deaths, we think this potential bias does not significantly change our conclusions.

\section{Conclusions}

The HSMR model for the Netherlands was sensitive to adjustment for frequency of readmissions. Model 1, without adjustment, compared to model 2, with adjustment, produced substantially different HSMR outcomes. The uncertainty introduced by these differences exceeded the uncertainty indicated by the $95 \%$ confidence intervals. Since model 2 turned out to be more accurate compared to model 1 with regard to c-statistics, goodness of fit and explanatory power, the authors would prefer to apply adjustment for frequency of readmission. Parties, also in other countries, making use of HSMR-calculations may decide for themselves whether or not it is clinically relevant in their situation to include frequent admissions in the model. In case of adjustment, a review period of at least three years is advisable.

\section{Author details}

${ }^{1}$ St. Antonius Hospital, P.O. Box 2500, 3430, EM Nieuwegein, the Netherlands. ${ }^{2}$ NIVEL, the Netherlands Institute for Health Services Research, P.O. Box 1568, Utrecht, the Netherlands. ${ }^{3}$ VU University Medical Centre, De Boelelaan 1117, 1081, HV Amsterdam, the Netherlands.

\section{Authors' contributions}

All authors have contributed significantly to the conception and design of the study. WB collected and analysed the data and produced the first draft of the manuscript. PS conducted the regression calculations. All authors revised the manuscript critically for important intellectual content and gave final approval of the version to be published.

\section{Competing interests}

The authors declare that they have no competing interests.

Received: 1 September 2011 Accepted: 4 April 2012

Published: 4 April 2012

\section{References}

1. Jarman B, Pieter D, van der Veen AA, et al: The hospital standardised mortality ratio: a powerful tool for Dutch hospitals to assess their quality of care? Qual Saf Health Care 2010, 19:9-13.

2. The 2010 Dr Foster Hospital Guide. [http://www.drfosterhealth.co.uk], (accessed 12 May 2011).

3. Lilford $\mathrm{R}$, Pronovost $\mathrm{P}$ : Using hospital mortality rates to judge hospital performance: a bad idea that just won't go away. BMJ 2010, 340:c2016.

4. Black N: Assessing the quality of hospitals. BMJ 2010, 340:c2066.

5. Penfold RB, et al: Do hospital standardised mortality ratios measure patient safety? HSMRs in the Winnipeg Regional Health Authority. Healthcare Papers 2008, 8(4):8-23.

6. Van den Bosch WF, Roozendaal KJ, Silberbusch J, Wagner J: Variation in coding patient data impacts hospital standardised mortality ratios (HSMR). Ned Tijdschr Geneeskd 2010, 154:A1189, in Dutch.

7. Van den Bosch WF, Graafmans WC, Pieter D, Westert GP: Cardiac centres and the HSMR. The impact of special medical procedures upon the hospital standardised mortality ratio. Ned Tijdschr Geneeskd 2008, in Dutch:1221-7, in Dutch.

8. Mohammed MA, Deeks JJ, Girling A, Rudge G, Carmalt M, Stevens AJ, Lilford RJ: Evidence of methodological bias in hospital standardised mortality ratios: retrospective database study of English hospitals. BMJ 2009, 338:b780.

9. Lilford R, Mohammed MA, Spiegelhalter D, Thomson R: Use and misuse of process and outcome data in managing performance of acute medical care: avoiding institutional stigma. Lancet 2004, 363:1147-1154.

10. Van den Bosch WF, Kelder HC, Wagner C: Predicting hospital mortality among frequently readmitted patients: HSMR biased by readmission. BMC Health Serv Res 2011, 11:57.

11. Bottle A, Jarman B, Aylin P: Hospital standardized mortality ratios: sensitivity analysis on the impact of coding. Heal Serv Res, doi:10.1111/ j.1475-6773.2011.01295.x. 
12. Clinical Classifications Software (CCS) for ICD-9-CM Fact Sheet. [http:// www.hcup-us.ahrq.gov/toolssoftware/ccs/ccsfactsheet.jsp\#what].

13. Hosmer DW, Lemeshow S: Applied Logistic Regression New York: Wiley; 2000, ISBN 0471615536.

14. Drye EE, Normand SLT, Wang Y, et al: Comparison of hospital riskstandardized mortality rates calculated by using in-hospital and 30-day models: an observational study with implications for hospital profiling. Ann Intern Med 2012, 156(no. 1 Part 1):19-26.

\section{Pre-publication history}

The pre-publication history for this paper can be accessed here: http://www.biomedcentral.com/1472-6963/12/91/prepub

doi:10.1186/1472-6963-12-91

Cite this article as: van den Bosch et al:: Variations in hospital standardised mortality ratios (HSMR) as a result of frequent readmissions. BMC Health Services Research 2012 12:91.

\section{Submit your next manuscript to BioMed Central} and take full advantage of:

- Convenient online submission

- Thorough peer review

- No space constraints or color figure charges

- Immediate publication on acceptance

- Inclusion in PubMed, CAS, Scopus and Google Scholar

- Research which is freely available for redistribution

Submit your manuscript at www.biomedcentral.com/submit 